\title{
Role of van der Waals forces in the diffraction of noble gases from metal surfaces
}

\author{
M. del Cueto, ${ }^{1}$ A. S. Muzas, ${ }^{1}$ G. Füchsel, ${ }^{2}$ F. Gatti, ${ }^{3}$ F. Martín,,${ }^{1,4,5,}{ }^{*}$ and C. Díaz ${ }^{1,5, \dagger}$ \\ ${ }^{1}$ Departamento de Química, Módulo 13, Universidad Autónoma de Madrid, 28049 Madrid, Spain \\ ${ }^{2}$ Leiden Institute of Chemistry, Gorlaeus Laboratories, Leiden University, P.O. Box 9502, 2300 RA Leiden, The Netherlands \\ ${ }^{3}$ CTMM, Institut Charles Gerhardt, UMR 5253, Université de Montpellier II, Place Eugène Bataillon, 34095 Montpellier, France \\ ${ }^{4}$ Instituto Madrileño de Estudios Avanzados en Nanociencia (IMDEA-Nanociencia), Cantoblanco 28049 Madrid, Spain \\ ${ }^{5}$ Condensed Matter Physics Center (IFIMAC), Universidad Autónoma de Madrid, 28049 Madrid, Spain \\ (Received 8 October 2015; revised manuscript received 26 January 2016; published 8 February 2016)
}

\begin{abstract}
The role of van der Waals (vdW) forces in the description of scattering processes of noble gases from metal surfaces is currently under debate. Although features of the potential energy surface such as anticorrugation or adsorption energies are sometimes found to be well described by standard density functional theory (DFT), the performance of DFT to describe diffraction spectra may rely on the accuracy of the vdW functionals used. To analyze the precise role of these vdW forces in noble gas diffraction by metal surfaces, we have thoroughly studied the case of $\mathrm{Ne} / \mathrm{Ru}(0001)$, for which accurate experimental results are available. We have carried out classical and quantum dynamics calculations by using DFT-based potentials that account for the effect of vdW interactions at different levels of accuracy. From the comparison of our results with experimental data, we conclude that the inclusion of vdW effects is crucial to properly describe diffraction of noble gases from metal surfaces. We show that among the vdW-DFT functionals available in the literature, not all of them can be used to accurately describe this process.
\end{abstract}

DOI: 10.1103/PhysRevB.93.060301

The diffraction of noble gases is largely used in surface science as a nondestructive analytical tool to investigate, for example, surface morphology and surface phonons (see Refs. [1,2] and refs. therein). Furthermore, this tool can also be used to study the dynamics of adsorbate/surface systems $[3,4]$. In order to extract the maximum amount of information from experimental diffraction spectra, a detailed comparison with theoretical simulations is often desirable. However, from a theoretical point of view, the description of the electronic structure of noble-gas atom/surface systems, in particular when metal surfaces are involved, is not a trivial matter due to the possible prominent role of van der Waals $(\mathrm{vdW})$ interactions. The first theoretical approach to treat these kind of systems was reported in the early 80's by Esbejerg and Nørskov [5], who proposed the use of an interaction potential proportional to the unperturbed electron density of the substrate at the position of the atomic projectile. But this approach was questioned only two years later by experimental results showing anticorrugation effects in He scattering from Ni(110) [6] that could not be reproduced with this simple model. Later on, in the 90's, first principles calculations were performed using a jellium model to describe the substrate $[7,8]$. Although this simple model is good enough to reproduce some general properties, to take into account the lattice structure is essential to analyze many other properties such as the corrugation amplitudes of the system, which are responsible for diffraction scattering phenomena.

The periodic lattice structure of a noble-gas atom/surface system can be well described by density functional theory (DFT) with periodic boundary conditions. However, standard DFT functionals do not include, per se, the effect of the van

\footnotetext{
*fernando.martin@uam.es

†cristina.diaz@uam.es
}

der Waals forces. Despite this fact, it has been already shown that standard DFT calculations, within the generalized gradient approximation (GGA), are able to account, for example, for the anticorrugation observed in the scattering of $\mathrm{He}$ atoms from several transition metals $[9,10]$. A recent study of adsorption of Xe on metal surfaces [11] suggests that standard DFT may be suitable to describe physisorption energies of noble gases on surface. However, this is arguable as standard functionals do not account for $\mathrm{vdW}$ interactions, which are expected to play some role in noble gas/surface interactions. It has been even suggested that standard DFT calculations might also provide an appropriate description of scattering of $\mathrm{He}$ and $\mathrm{Ne}$ from a metal surface, due to the small interatomic distances at the classical turning point [11]. However, to our knowledge, this hypothesis has never been tested by performing a direct comparison with experimental diffraction spectra obtained for the same system and by using exactly the same incidence conditions. Recent theoretical results [12,13] suggest that the inclusion of $\mathrm{vdW}$ interactions is indeed important to describe the diffraction of $\mathrm{He}$ on $\mathrm{MgO}$ (insulator) surfaces.

In this paper we present a detailed theoretical study of diffraction of noble-gas atoms from metal surfaces, based on state-of-the-art DFT calculations, with explicit inclusion of $\mathrm{vdW}$ effects. We focus on the scattering of $\mathrm{Ne}$ as it shows a higher sensitivity to structural details of the surface than $\mathrm{He}$, and experimentally derived corrugations amplitudes are typically twice as large as those of $\mathrm{He}$ [14-16]. Indeed, due to its higher sensitivity, Ne diffraction can be used to probe structural details of surfaces consisting of different types of atoms. For example, scattering experiments of $\mathrm{Ne}$ from $\mathrm{NiAl}(110)$ [17] reveal the presence of the two types of atoms, $\mathrm{Ni}$ and $\mathrm{Al}$, whereas diffraction of $\mathrm{He}$ only reveals one of them depending on the specific incidence conditions. Also, in the case of $\mathrm{Ni}(110)$ [14], $\mathrm{Cu}(110)$ [15], and, more recently, $\mathrm{Ru}(0001)$ surfaces [18], diffraction by $\mathrm{Ne}$ leads to 
richer diffraction spectra than those from He. In this Rapid Communication, we have chosen the latter $\mathrm{Ru}(0001)$ surface to perform our systematic theoretical study.

For this, we have made use of the methods already available in the literature (see Refs. [19-22] and Refs. therein) that incorporate vdW forces in DFT electronic structure calculations, either through novel exchange-correlation functionals or as additional potential terms in the Kohn-Sham Hamiltonian. In surface science, these methods have been rather successfully used in describing multilayer systems involving graphene and boron-nitride [23], organic molecules adsorbed on metal [24-37] and nonmetal surfaces [38-40], graphene adsorbed on metal surfaces [41-43], and even organic molecules adsorbed on graphene adsorbed on metal surfaces [44]. These vdW methods have also been used to study the interaction of polyatomic [45-47] and diatomic[48-50] molecules with surfaces, and even with aromatic systems [51]. However, to the best of our knowledge, so far they have not been used to study the diffraction of noble gases from metal surfaces. Here we show that not only inclusion of $\mathrm{vdW}$ interactions is essential to properly describe this process, but also that not all available methods are able to do it accurately, despite their remarkable performance in other contexts.

To perform our study, we have worked within the BornOppenheimer static surface (BOSS) approximation. The use of the BO approximation is justified by the low incidence energy $\left(\mathrm{E}_{i}\right)$ of the projectile. The SS approximation is a reasonable assumption in view of the low surface temperature at which the experiments are carried out, around $100 \mathrm{~K}$. Within the BOSS approximation, we first compute a continuous potential energy surface (PES), and then use it to perform both classical and quantum dynamics. To build each of the five PESs used in our study, we have applied the corrugation reducing procedure (CRP) [52] to a set of DFT data. DFT electronic structure calculations have been computed using the plane wave based code VASP [53-56]. In these calculations, the electron-ion interaction is described through a projector augmented wave (PAW) [57,58]. To describe the exchange-correlation energy of the electrons, we have applied the generalized gradient approximation in two different ways: (i) by using the PBE functional for exchange and correlation [59] plus Grimme's corrections $[60,61] \mathrm{PBE}+\mathrm{D} 2$ and $\mathrm{PBE}+\mathrm{D} 3$ to account for dispersion forces (in Grimme's approach the effect of the vdW forces is taken into account by adding a correction term to the Kohn-Sham DFT energy), and the, here called, PBE+vdW ${ }^{S}$ functional developed by Ruiz et al. [62], which includes the many-body collective response of substrate electrons, the so-called screening; (ii) by using vdW-DF functionals, developed originally by Lundqvist et al. [63], which include the effect of dispersion forces through a nonlocal electron correlation function that is added to the usual exchangecorrelation functional. In the latter case, we have tested the optB86b-DF (hereafter called vdW-DF) $[64,65]$ and the vdW-DF2 functionals [66]. To model the adsorbate/substrate systems, we have used a five-layer slab and a $(2 \times 2)$ surface unit cell. To avoid spurious results on the $Z$ direction due to the imposed periodicity, a vacuum layer of $20 \AA$ has been used. The first Brillouin zone has been sampled using $11 \times 11 \times 1$ Gamma centered $k$ points. A cutoff energy of $700 \mathrm{eV}$ has been set for the plane wave basis.

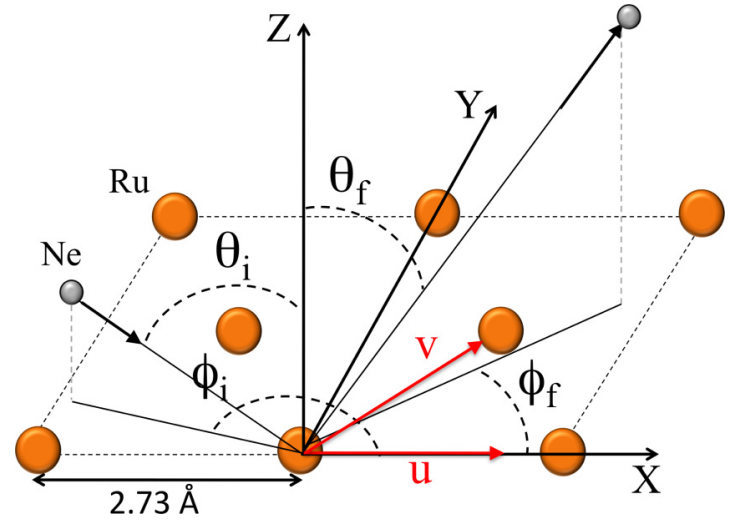

FIG. 1. Cartesian $(X, Y, Z)$ and crystal $(u, v)$ coordinate systems.

The dynamical calculations have been performed by using both classical and quantum methods. The classical trajectory (CT) method allows us to explore the PES and to get a simple dynamical picture of the scattering process. It also facilitates the determination of the dynamically relevant regions of the PES. A classical trajectory is computed by solving the Hamilton classical equations of motion. In order to ensure low statistical errors, we have run of the order of $2 \times 10^{5}$ trajectories for each incidence energy $\left(\mathrm{E}_{i}\right)$ and incidence angles $\left(\theta_{i}, \phi_{i}\right)$-see Fig. 1 for the definition of coordinates. Quantum calculations have been performed by using the multiconfiguration time-dependent Hartree (MCTDH) method [67-69], more precisely the Heidelberg MCTDH package [70]. In the MCTDH method, the nuclear wave function of the system is written as a sum of products of single-particle functions (SPFs), in such a way that both expansion coefficients and the SPFs are optimized by a variational principle and are time dependent. Finally, the SPFs are represented by linear combinations of time-independent primitive basis functions (plane waves). A fast Fourier transform (FFT) representation is used for each of the three degrees of freedom. Using MCTDH, diffraction probabilities are obtained through a flux analysis of the reflected wave function upon absorption by a complex absorbing potential, which is placed in the noninteracting $Z$ region. The parameters used in the MCTDH calculations are listed in Table I.

In Fig. 2 we display the classical turning points for three different experimental conditions [18] and for all the DFT functionals used in this Rapid Communication to build the different PESs. This figure shows that the classical turning point (CTP) barely depends on the incidence conditions in the angular and energy ranges considered in this work, but varies considerably with the underlying DFT functional used. As expected, functionals including vdW forces attract the atoms towards the surface (smaller value of the CTP). The $\mathrm{PBE}+\mathrm{D} 2 \mathrm{PES}$ is the most attractive one and the PBE PES the least of all of them. Atoms moving on the PBE+D3, vdW-DF, vdW-DF2 and PBE+vdW ${ }^{S}$ PESs explore similar regions of their corresponding PESs. Features expected to have an important effect on the diffraction spectra, which will also depend on the DFT functional used to describe the atom surface interaction, are the topology and shape of the PES. In Fig. 3, we have plotted 2D PESs along $u$ and $v$ coordinates 
TABLE I. MCTDH calculations parameter as a function of the initial incidence conditions. $\mathrm{E}_{i}$ is the overall total energy of the wave packet, $\mathrm{N}_{u, v}$ and $\mathrm{N}_{Z}$ are the FFT primitive points/functions for coordinates $u, v$, and $Z$, respectively.

\begin{tabular}{cc}
\hline \hline $\mathrm{E}_{i}=43 \mathrm{meV}$ & $\mathrm{E}_{i}=64 \mathrm{meV}$ \\
$\theta_{i}=45^{\circ}$ & $\theta_{i}=47^{\circ}$ \\
$\phi_{i}=30^{\circ}$ & $\phi_{i}=30^{\circ}$
\end{tabular}

Initial wave packet

Width, $\Delta Z_{0}(\AA)$

Position, $Z_{0}(\AA)$

$u^{*}$ and $v^{*}$ Momentum (a.u.)

$\begin{array}{cc}0.4 & 0.4 \\ 8.5 & 8.5 \\ 6.6978 & 8.2210 \\ -7.6214 & -8.9679\end{array}$

$Z$ Momentum (a.u.)

FFT

Type $u, v, Z$

$\begin{array}{cc}\text { FFT } & \text { FFT } \\ 0.0-10.92 & 0.0-10.92 \\ 720 & 720 \\ -0.75-15.00 & -0.75-15.00 \\ 324 & 324\end{array}$

Complex absorbing potential

Z-range $(\AA)$

$\begin{array}{cc}8.5-15.0 & 8.5-15.0 \\ 4.5786 \times 10^{-6} & 4.5786 \times 10^{-6} \\ 10 & 10 \\ 4000-5000 & 4000-5000\end{array}$

SPFs per degree of freedom

Propagation time (fs)

$4000-5000$

$4000-5000$

*rystal coordinate (see Fig. 1).

at the CTP obtained from all five DFT functionals. A closer inspection of the PESs region from which the atoms scatter (back) reveals some interesting characteristics. (i) We observe anticorrugation (i.e., a more repulsive potential for the hollow than for the top site) for all PES except for the PBE+D2 one. At first sight, this result seems to be in contradiction with previous experimental and theoretical results [9,14], which showed normal corrugation for $\mathrm{Ne}$ diffraction from $\mathrm{Ni}$ and $\mathrm{Rh}$ surfaces. In this respect, it is important to point out that all our PESs do exhibit normal corrugation in regions close to the ruthenium surface, although these regions are dynamically inaccessible at the incidence energies considered in this Rapid

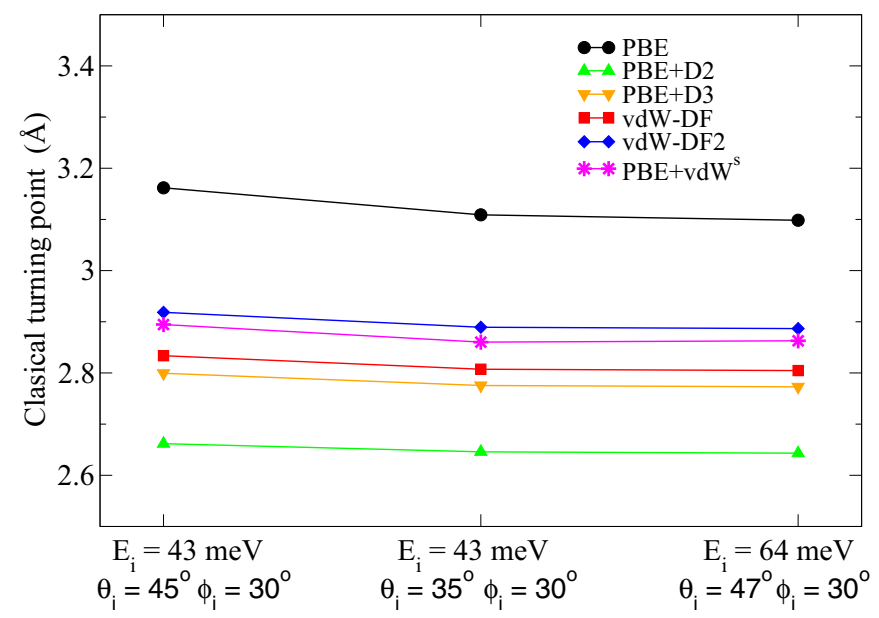

FIG. 2. Average classical turning points (CTP) as a function of the DFT functional used to build the PES, for several experimental incidence conditions.
Communication. In fact, we have checked that at higher $E_{i}$ than those considered in Ref. [18], and in this work, the Ne atoms would feel normal corrugation instead of anticorrugation. (ii) The PBE+D2 PES, the only one showing normal corrugation (even in the attractive region of the PES), is by far the most corrugated one-defining corrugation as the difference
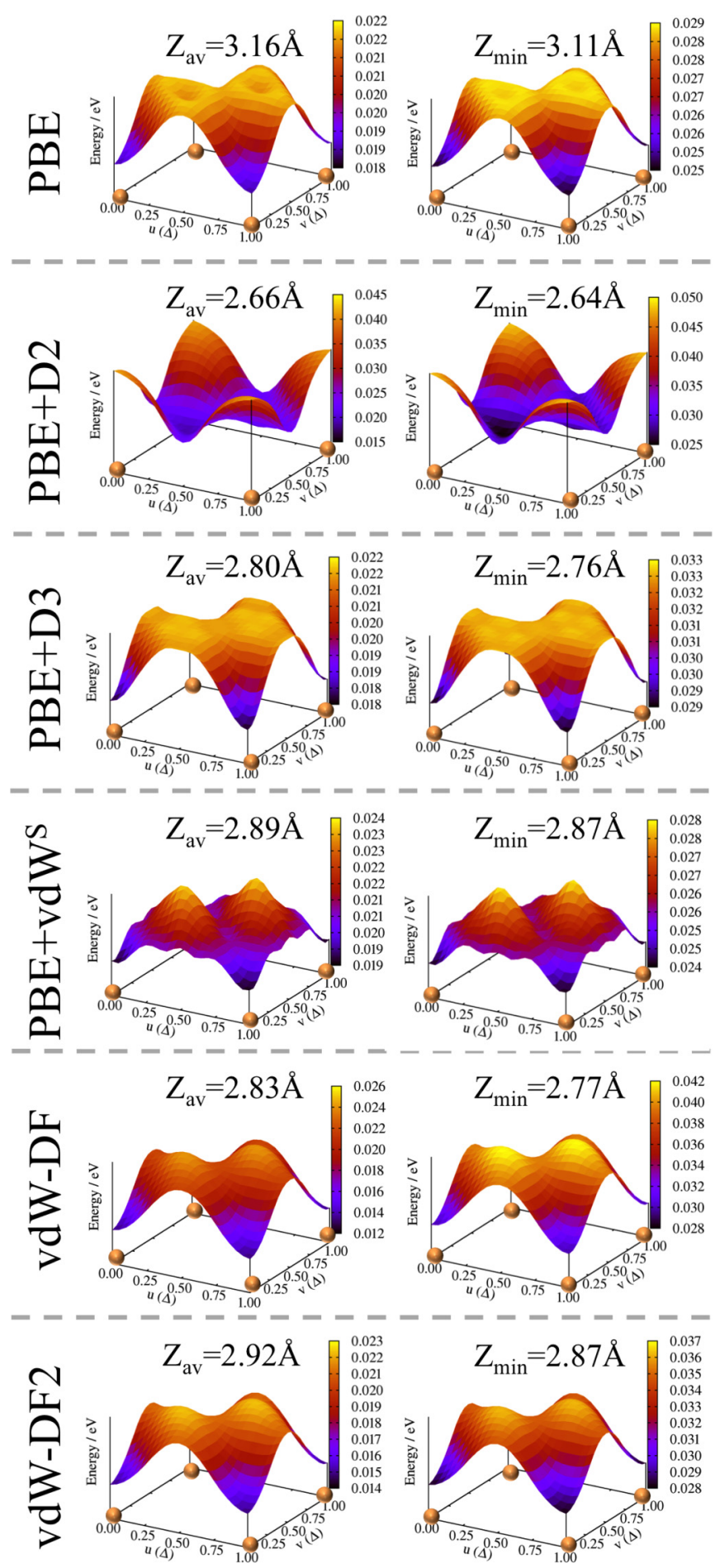

FIG. 3. $(u, v)$ Electronic landscape at the corresponding average and minimum CTP found in our classical dynamics simulations. Orange spheres indicate the position of the $\mathrm{Ru}$ atoms in the unit cell. $\Delta=2.73 \AA$. 


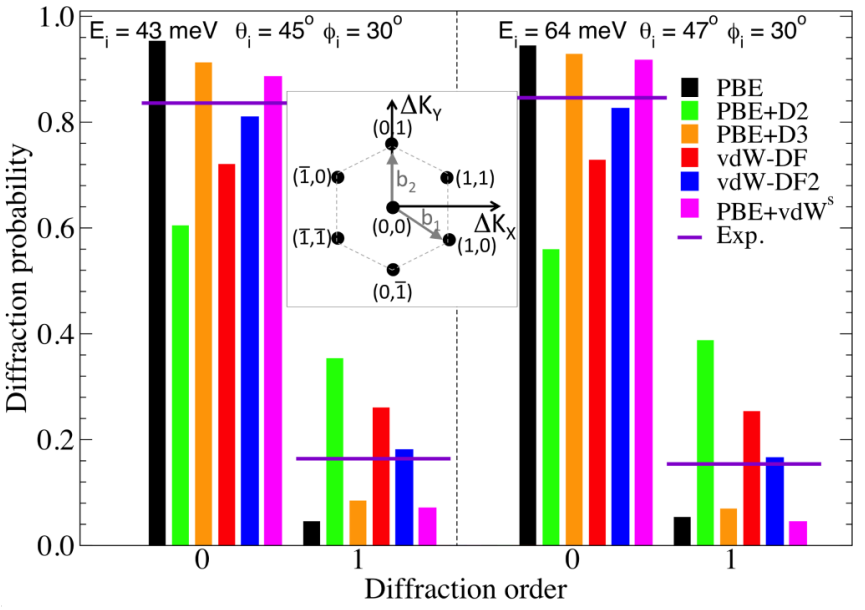

FIG. 4. Diffraction order probabilities for $\mathrm{Ne} / \mathrm{Ru}(0001)$ under several experimental incidence conditions. Solid bars: Quantum dynamics simulations; violet horizontal lines: Experimental data taken from Ref. [18]. The inset shows the reciprocal lattice for $\mathrm{Ru}(0001)$.

between top and hollow potentials. In fact, our results indicate that the DFT-D2 approach not only shifts the whole repulsive part of the PBE PES downwards, but it also affects it in a complex way. The PBE and DFT+D3 PESs exhibit very small corrugation, whereas the corrugation displayed by the vdW-DF and vdW-DF2 PESs is about two and three times larger, respectively, than that observed for the PBE PES. The $\mathrm{PBE}+\mathrm{vdW} \mathrm{W}^{S} \mathrm{PES}$ exhibits a corrugation slightly larger than the $\mathrm{DFT}+\mathrm{D} 3$ one.

From the different characteristics of the calculated PESs, one can already anticipate that diffraction intensities will be very different. Now the question is which one, if any, can yield accurate diffraction intensities. To answer this question, we have plotted zeroth and first order diffraction probabilities in Fig. 4, for two sets of experimental incidence conditions. From a comparison with experimental results we can already see that PBE, PBE+D3, and PBE+vdW ${ }^{S}$ PESs substantially underestimate diffraction. In the case of the PBE+D2 PES, measured diffraction probabilities are largely overestimated. The best agreement with experiment is obtained with the vdW-DF and vdW-DF2 PESs. In the case of the vdW-DF2 calculations, we observe, similar to a previous work for more complex molecule/surface systems [71], that the strength of the asymptotic vdW attraction is less than a half compared to the one obtained from the vdW-DF calculations. But long range effects close to the asymptotic region $(Z>7 \AA)$ seem to play a minor role in the case of atomic diffraction.

However, an accurate PES should not only yield good total diffraction intensities, but also accurate diffraction peak probabilities. Thus, we have evaluated the individual diffraction peak intensities for each of the four first-order peaks observed experimentally. The results are plotted in Fig. 5, and show that experimental trends and peak intensities can be fairly well reproduced with the vdW-DF PES, but even better with the vdW-DF2 PES. Discrepancies between experiment and theory may arise from the experimental surface temperature $(100 \mathrm{~K})$, which may lead to lower peak intensities. Therefore,

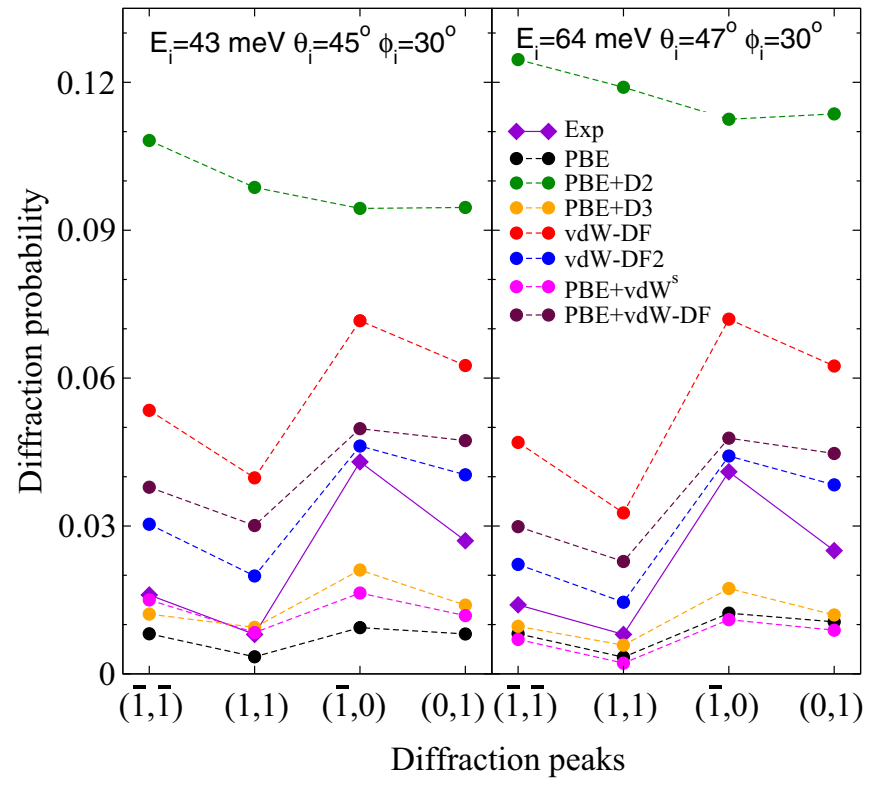

FIG. 5. First order diffraction peak probabilities for $\mathrm{Ne} / \mathrm{Ru}(0001)$. Dashed lines with symbols: Quantum dynamics simulations; solid lines with symbols: Experimental results taken from Ref. [18].

the quantitative better agreement obtained with the vdW-DF2 functional should not be taken as a conclusive proof that this functional is more appropriate than the vdW-DF one to account for dispersion forces, in gas noble atom diffraction phenomena. They both give qualitatively good results. On the other hand, $\mathrm{PBE}+\mathrm{D} 3$ and $\mathrm{PBE}+\mathrm{vdW} \mathrm{W}^{S}$ underestimate diffraction probabilities and barely reproduce experimental trends. Although $\mathrm{PBE}+\mathrm{D} 3$ and $\mathrm{PBE}+\mathrm{vdW}^{S}$ PESs yield similar first order diffraction probabilities, the corrugation felt by the projectiles is rather different. For the $\mathrm{PBE}+\mathrm{vdW}^{S} \mathrm{PES}$, we have also found non-negligible second order diffraction probabilities that are not observed in the experiment. The PES+D2 results largely overestimate experimental peak intensities and deviate considerably from the experimental trends. This holds true for all considered incident conditions. At this point, we note that the PBE+vdW functional has been found to accurately describe, for example, the adsorption of heavy noble atoms and organic molecules on metal surfaces [62,72], whereas PBE-D functionals do not. However, our results suggest that the description of diffraction peaks, which rely on the proper description of the surface corrugation, is still a challenge for pairwise-based correction methods. Here, it is important to point out that diffraction probabilities obtained by coupling the vdW-DF dispersion correction with the PBE functional (denoted PBE+vdW-DF in Fig. 5) are in good agreement with the vdW-DF and vdW-DF2 ones, as well as with the experimental results (see Fig. 5). This further supports our conclusion that a proper description of $\mathrm{vdW}$ forces, that goes beyond pairwise-based corrections, is a requisite for an accurate description of noble-gas diffraction from metal surfaces.

In summary, we have performed a systematic theoretical study of $\mathrm{Ne}$ diffractive scattering from a $\mathrm{Ru}(0001)$ metal surface using 3D DFT-based potential energy surfaces (PESs), 
in which the effect of van der Waals dispersion forces is taken into account. We have used seven of these PESs, which have been obtained by one of the following two different approaches, successfully used in the past to study molecule/surface systems: in the first approach, the van der Waals dispersion energy is added to the DFT energy [60-62], and, in the second one, a nonlocal correction term is added to the usual Kohn-Sham exchange-correlation functional $[65,66]$. Using these PESs we have carried out classical and quantum dynamics calculations, showing that the inclusion of $\mathrm{vdW}$ forces is essential to accurately reproduce measured diffraction spectra. Interestingly, among the vdW corrections considered in this work, we have found that the vdW-DF [65] functional performs qualitatively well, while almost quantitative agreement with experiment is obtained by using the vdW-DF2 [66] functional. Our analysis suggests that the PES regions probed by the atomic beam exhibit relative small amplitudes $(\approx 10 \mathrm{meV})$ and anticorrugation. To the best of our knowledge, anticorrugation effects have only been described previously in the case of He diffraction from metal surfaces.

We hope that the present study will inspire new theoretical work on diffraction of noble gases by metal surfaces. These systems have been widely studied from an experimental point of view (see Ref. [1] and refs. therein), but accurate theoretical analyses are still in their infancy $[7,73]$.

\section{ACKNOWLEDGMENTS}

We thank BSC-RES and CCC-UAM for allocation of computer time. Work partially supported by the MICINN Projects No. FIS2013-42002-R and No. CTQ2013-50150EXP, and the CAM Project No. S2013/MIT-2850. C. Díaz acknowledges the Ramón y Cajal program, M. del Cueto and A. S. Muzas the FPI program of the MICINN, and G. Füschel thanks CW-NWO for financial support through a TOP grant.
[1] D. Farías and K. H. Rieder, Rep. Prog. Phys. 61, 1575 (1998).

[2] A. P. Graham, Surf. Sci. Rep. 49, 115 (2003).

[3] G. Alexandrowicz, A. P. Jardine, P. Fouquet, S. Dworski, W. Allison, and J. Ellis, Science 304, 1790 (2004).

[4] G. Alexandrowicz, A. P. Jardine, P. Fouquet, S. Dworski, W. Allison, and J. Ellis, Phys. Rev. Lett. 93, 156103 (2004).

[5] N. Esbjerg and J. K. Norskov, Phys. Rev. Lett. 45, 807 (1980).

[6] K. H. Rieder and N. Garcia, Phys. Rev. Lett. 49, 43 (1982).

[7] J. Ellis, K. Hermann, F. Hofmann, and J. P. Toennies, Phys. Rev. Lett. 75, 886 (1995).

[8] F. Montalenti, M. I. Trioni, G. P. Brivio, and S. Crampi, Surf. Sci. 364, L595 (1996).

[9] M. Petersen, S. Wilke, P. Ruggerone, B. Kohler, and M. Scheffler, Phys. Rev. Lett. 76, 995 (1996).

[10] N. Jean, M. I. Trioni, G. P. Brivio, and V. Bortolani, Phys. Rev. Lett. 92, 013201 (2004).

[11] J. L. F. DaSilva, C. Stampfl, and M. Scheffler, Phys. Rev. Lett. 90, 066104 (2003).

[12] R. Martinez-Casado, G. Mallia, D. Usvyat, L. Maschio, S. Casassa, M. Schütz, and N. M. Harrison, Phys. Chem. Chem. Phys. 13, 14750 (2011).

[13] R. Martinez-Casado, D. Usvyat, G. Mallia, L. Maschio, S. Casassa, J. Ellis, M. Schütz, and N. M. Harrison, Phys. Chem. Chem. Phys. 16, 21106 (2014).

[14] K. H. Rieder and W. Stocker, Phys. Rev. Lett. 52, 352 (1984).

[15] B. Salanon, J. Phys. 45, 1373 (1984).

[16] G. P. Brivio and M. I. Trioni, Rev. Mod. Phys. 71, 231 (1999).

[17] D. Farías, M. Patting, and K. H. Rieder, J. Chem. Phys. 117, 1797 (2002).

[18] M. Minniti, C. Díaz, J. L. Fernández-Cunado, A. Politano, D. Maccariello, F. Martín, and R. Miranda, J. Phys. Condens. Matter 24, 354002 (2012).

[19] A. Tkatchenko, L. Romaner, O. T. Hofmann, E. Zojer, C. Ambrosch-Draxl, and M. Scheffler, MRS Bull. 35, 435 (2010).

[20] S. Grimme, WIREs: Comput. Mol. Sci. 1, 211 (2011).

[21] J. Klimes, and A. Michaelides, J. Chem. Phys. 137, 120901 (2012).
[22] K. Berland, V. R. Cooper, K. Lee, E. Schröfer, T. Thonhauser, P. Hyldgaard, and E. I. Lundqvist, Rep. Prog. Phys. 78, 066501 (2015).

[23] W. Gao and A. Tkatchenko, Phys. Rev. Lett. 114, 096101 (2015).

[24] K. Berland, T. L. Einstein, and P. Hyldgaard, Phys. Rev. B 80, 155431 (2009).

[25] L. Romaner, D. Nabok, and P. Puschnig, New J. Phys. 11, 053010 (2009).

[26] D. Sun and et al., Phys. Rev. B 82, 201410 (2010).

[27] K. Lee, Y. Morikawa, and D. C. Langreth, Phys. Rev. B 82, 155461 (2010).

[28] K. Toyada, I. Hamada, K. Lee, S. Yanagisawa, and Y. Morikawa, J. Chem. Phys. 132, 134703 (2010).

[29] M. Mura, A. Culans, T. Thonhauser, and L. Kantorovich, Phys. Chem. Chem. Phys. 12, 4759 (2010).

[30] G. Mercurio and et al., Phys. Rev. Lett. 104, 036102 (2010).

[31] J. Wyrick and et al., Nano Lett. 11, 2944 (2011).

[32] W. Liu, J. Carrasco, B. Santra, A. Michaelides, M. Scheffler, and A. Tkatchenko, Phys. Rev. B 86, 245405 (2012).

[33] G. Li, I. Tamblyn, V. R. Cooper, H. J. Gao, and J. B. Neaton, Phys. Rev. B 85, 121409 (2012).

[34] H. Yildirim, T. Greber, and A. Kara, J. Phys. Chem. C 117, 20572 (2013).

[35] K. H. Dostert, C. P. O’Brien, W. Riedel, A. Savara, W. Liu, M. Oehzelt, A. Tkatchenko, and S. Schauermann, J. Phys. Chem. C 118, 27833 (2014).

[36] C. Wagner, N. Fournier, V. G. Ruiz, C. Li, K. Müller, M. Rohlfing, A. Tkatchenko, T. Temiroy, and F. S. Tautz, Nat. Commun. 5, 5568 (2014).

[37] W. Liu, F. Maaß, M. Wollenbockel, C. Bronner, M. Schulze, S. Soubatch, F. S. Tautz, P. Tegeder, and A. Tkatchenko, Phys. Rev. Lett. 115, 036104 (2015).

[38] S. D. Chakarova-Kack, E. Schröder, B. I. Lundqvist, and D. C. Langreth, Phys. Rev. Lett. 96, 146107 (2006).

[39] K. Johnston, J. Kleis, B. I. Lundqvist, and R. M. Nieminen, Phys. Rev. B 77, 121404 (2008).

[40] G. Li, V. R. Cooper, J. H. Cho, S. Du, H. J. Gao, and Z. Zhang, Phys. Rev. B 84, 241406 (2011). 


\section{DEL CUETO et al.}

[41] C. Busse, P. Lazic, R. Djemour, J. Coraux, T. Gerber, N. Atodiresei, V. Caciuc, R. Brako, A. T. N'Diaye, S. Blügel, J. Zegenhagen, and T. Michely, Phys. Rev. Lett. 107, 036101 (2011).

[42] D. Stradi, S. Barja, C. Díaz, M. Garnica, B. Borca, J. J. Hinarejos, D. Sánchez-Portal, M. Alcamí, A. Arnau, A. L. Vázquez de Parga, R. Miranda, and F. Martín, Phys. Rev. Lett. 106, 186102 (2011).

[43] I. Loncaric and V. Despoja, Phys. Rev. B. 90, 075414 (2014).

[44] M. Garnica, D. Stradi, S. Barja, F. Calleja, C. Díaz, M. Alcamí, N. Martín, A. L. Vázquez de Parga, F. Martín, and R. Miranda, Nat. Phys. 9, 368 (2013).

[45] X. Li, J. Feng, E. Wang, S. Meng, J. Klimeš, and A. Michaelides, Phys. Rev. B 85, 085425 (2012).

[46] J. Carrasco, J. Klimes, and A. Michaelides, J. Phys. Chem. 138, 024708 (2013).

[47] G. Tocci, L. Joly, and A. Michaelides, Nano Lett. 14, 6872 (2014).

[48] X. Ren, P. Rinke, and M. Scheffler, Phys. Rev. B 80, 045402 (2009).

[49] M. Wijzenbroek and G. J. Kroes, J. Chem. Phys. 140, 084702 (2014).

[50] L. Martín-Gondre, J. I. Juaristi, M. Blanco-Rey, R. Díez-Muiño, and M. Alducin, J. Chem. Phys. 142, 074704 (2015).

[51] C. Díaz, Theor. Chem. Acc. 134, 105 (2015).

[52] H. F. Busnengo, A. Salin and W. Dong, J. Chem. Phys. 112, 7641 (2000).

[53] G. Kresse and J. Hafner, Phys. Rev. B 47, 558 (1993).

[54] G. Kresse and J. Hafner, Phys. Rev. B 49, 14251 (1994).

[55] G. Kresse and J. Furthmüller, Comput. Mater. Sci. 6, 15 (1996).

[56] G. Kresse and J. Furthmüller, Phys. Rev. B 54, 11169 (1996).

[57] P. E. Blochl, Phys. Rev. B 50, 17953 (1994).
PHYSICAL REVIEW B 93, 060301(R) (2016)

[58] G. Kresse and D. Joubert, Phys. Rev. B 59, 1758 (1999).

[59] J. P. Perdew, K. Burke, and M. Ernzerhof, Phys. Rev. Lett. 77, 3865 (1996).

[60] S. Grimme, J. Comput. Chem. 27, 1787 (2006).

[61] S. Grimme, J. Antony, S. Ehrlich, and S. Krieg, J. Chem. Phys. 132, 154104 (2010).

[62] V. G. Ruiz, W. Liu, E. Zojer, M. Scheffler, and A. Tkatchenko, Phys. Rev. Lett. 108, 146103 (2012).

[63] M. Dion, H. Rydberg, E. Schröder, D. C. Langreth, and B. I. Lundqvist, Phys. Rev. Lett. 92, 246401 (2004).

[64] J. Klimes, D. R. Bowler, and A. Michaelides, J. Phys.: Cond. Matt. 22, 022201 (2010).

[65] J. Klimes, D. R. Bowler, and A. Michaelides, Phys. Rev. B 83, 195131 (2011).

[66] K. Lee, E. D. Murray, L. Kong, B. I. Lundqvist, and D. C. Langreth, Phys. Rev. B 82, 081101 (2010).

[67] M. H. Beck, A. Jäckle, G. A. Worth, and H. D. Meyer, Phys. Rep. 324, 1 (2000).

[68] H. D. Meyer and G. A. Worth, Theor. Chem. Acc. 109, 251 (2003).

[69] Multidimensional Quantum Dynamics: Mctdh Theory and Applications, edited by H.-D. Meyer, F. Gatti, and G. A. Worth (Wiley-VCH, Weinheim, 2009).

[70] G. A. Worth, M. H. Beck, A. Jäckle, and H.-D. Meyer, The MCTDH Package, Version 8.1 (2000). H. -D. Meyer, Version 8.3 (2002), Version 3.4 (2007). See http://mctdh.uni-hd.de/ .

[71] J. Carrasco, W. Liu, A. Michaelides, and A. Tkatchenko, J. Chem. Phys. 140, 084704 (2014).

[72] W. Liu, A. Tkatchenko, and M. Scheffler, Acc. Chem. Res. 47, 3369 (2014).

[73] C. Huang, D. A. MacLaren, J. Ellis, and W. Allison, Phys. Rev. Lett. 96, 126102 (2006). 